\title{
An inductive power link for a wireless endoscope
}

\author{
Bert Lenaerts*, Robert Puers \\ Katholieke Universiteit Leuven, Department of ESAT-MICAS, Kasteelpark Arenberg 10, B-3001 Leuven, Belgium
}

Received 31 March 2006; received in revised form 30 May 2006; accepted 15 June 2006

Available online 14 August 2006

\begin{abstract}
An inductive link is presented that can handle freedom of motion. The envisaged application is to power a wireless camera capsule used for non-invasive visual inspection of the small bowel. Up to $150 \mathrm{~mW}$ of usable dc power can be delivered to the capsule for the entire duration of its travel along the gastric track. The outer dimensions of the power receiver compartment inside the capsule are $\emptyset 10 \mathrm{~mm} \times 13 \mathrm{~mm}$. The power efficiency of the link is measured to be $1 \%$ under worst-case geometrical conditions. The issue of patient's health and safety regarding exposure to the electromagnetic field is addressed. To this purpose, an experimental method is applied to predict specific absorption rates. Conversely, the effect of biological tissue on link performance and practical workability is evaluated as well. Electric shielding of the transmitter coil is applied to reduce biological tissue interaction. In this way, appliance on a living subject is made both safe and practically feasible.

(c) 2006 Elsevier B.V. All rights reserved.
\end{abstract}

Keywords: Wireless endoscopy; Capsule endoscopy; Transcutaneous link; Inductive powering; IVP

\section{Introduction}

A wireless endoscope, or swallowable camera capsule, is taken in orally whereupon it continues the natural way down the gastric track (Iddan et al., 2000). In the meantime a digital video stream is broadcasted, stored externally and used for diagnostic purposes afterwards. Wireless endoscopy allows the inspection of regions deep inside the body - more specifically the complete small bowel - that are not accessible with traditional push enteroscopy (Appleyard et al., 2000; Costamagna et al., 2002; Fireman et al., 2003).

Commercially existing camera capsules (Iddan et al., 2000) rely on batteries for power. The limited energy budget restricts the quality and amount of images that can be obtained. A novel wireless endoscopy concept, the Intracorporal Video Probe (IVP) (Arena et al., 2005), makes use of an inductive power link to overcome this limitation. The power consumption of this system while capturing a high-quality image is as high as $150 \mathrm{~mW}$. The outer dimensions of the IVP camera capsule are $\emptyset 11 \mathrm{~mm} \times 28 \mathrm{~mm}$. The volume inside of it assigned for power is $\varnothing 10 \mathrm{~mm} \times 13 \mathrm{~mm}$.

\footnotetext{
* Corresponding author.

E-mail addresses: bert.lenaerts@esat.kuleuven.be (B. Lenaerts), robert.puers@esat.kuleuven.be (R. Puers).
}

Commonly encountered inductive link designs assume a more or less fixed orientation and/or position of the coupled induction coils. In contrast with this is a wireless endoscope free to adopt any position or orientation within the abdominal volume. The power link presented here can cope with these degrees of freedom. It represents a novel solution to the more general problem of omnidirectional inductive powering.

\section{Link realization}

\subsection{Coupled coils}

The freedom of motion of the camera capsule imposes the use of multiple coils either externally or internally. The choice for a certain coil arrangement is a crucial step in an omnidirectional link design. Together with other geometrical aspects, it sets an upper limit on the link efficiency that can be achieved. This issue is dealt with extensively in previous work (Lenaerts and Puers, 2005). For the application of wireless endoscopy, three internal coils and only one external solenoid were found to yield the highest efficiency (Lenaerts and Puers, 2005).

Besides all geometric considerations, an electric optimization needs to be carried out, mostly dealing with resistive losses and impedance matching (Ko et al., 1977). This involves identification of the appropriate type of wire and number of turns. The 
coil designs used here are discussed in greater detail in preceding work (Lenaerts and Puers, 2005).

The electric optimization mentioned above has the frequency of operation as an important parameter. To find the optimal frequency, maximizing the link efficiency, is far from straightforward. Furthermore, besides the efficiency argument, other criteria exist for selecting the frequency of operation. As shown later in this text, human body absorption is very dependent upon frequency. In this respect, the optimal frequency would be the one that minimizes tissue dissipation while still reaching the power specifications. In line with the existing experience in the group (Schuylenbergh and Puers, 1996; Puers et al., 2000), a frequency of $1 \mathrm{MHz}$ was chosen as a starting point in a first prototype.

\subsection{Field generator}

An inverter drives a sinusoidal high-amplitude electric current through the primary coil at a frequency of approximately $1 \mathrm{MHz}$. The primary coil is installed around the patient's trunk (depicted in Supplementary Fig. 2). The inner diameter of the coil is $41 \mathrm{~cm}$. The coil length is $30 \mathrm{~cm}$. A rather homogeneous distribution of the magnetic field inside the trunk is achieved by the solenoid geometry. Rectangular Litz cable is used, drastically reducing resistive losses at higher frequencies (Sullivan, 1999).

A class E inverter topology was chosen (Sokal and Sokal, 1975). Fig. 1 depicts the design schematic. A pulse train $V_{\mathrm{p}}$ (period $947 \mathrm{~ns}$, duty cycle 0.4 ) is applied at the transistor gate. The resonance frequency of the tank constituted by $L_{1}$ and $C_{\text {tank }}$ is slightly higher than the pulse frequency $(1116 \mathrm{kHz}$ compared to $1056 \mathrm{kHz}$ ). In this way, the tank is effectively seen as a highly inductive impedance. Hence, the current supplied to the tank is relatively low while the current inside the tank can build up to high values, which is desired. The equivalent series resistance (ESR) $R_{1}$ accounts for the coil losses and determines the tank quality and current damping. Complementary to $C_{\text {tank }}$ that preserves the switch from high current amplitudes, it is $C_{\text {ser }}$ that shields the switch transistor from the high voltage over $L_{1}$. The amplitude of the tank current is proportional to the dc supply voltage $V_{\mathrm{CC}}$. The choke inductor $L_{\mathrm{choke}}$ blocks the ac current from the supply. Component values were calculated using the high- $Q$ approximation (Raab, 1977).

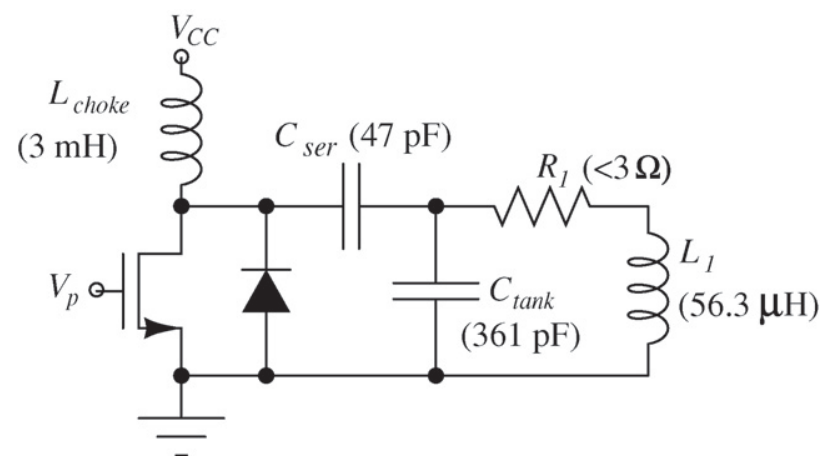

Fig. 1. Inverter design schematic.

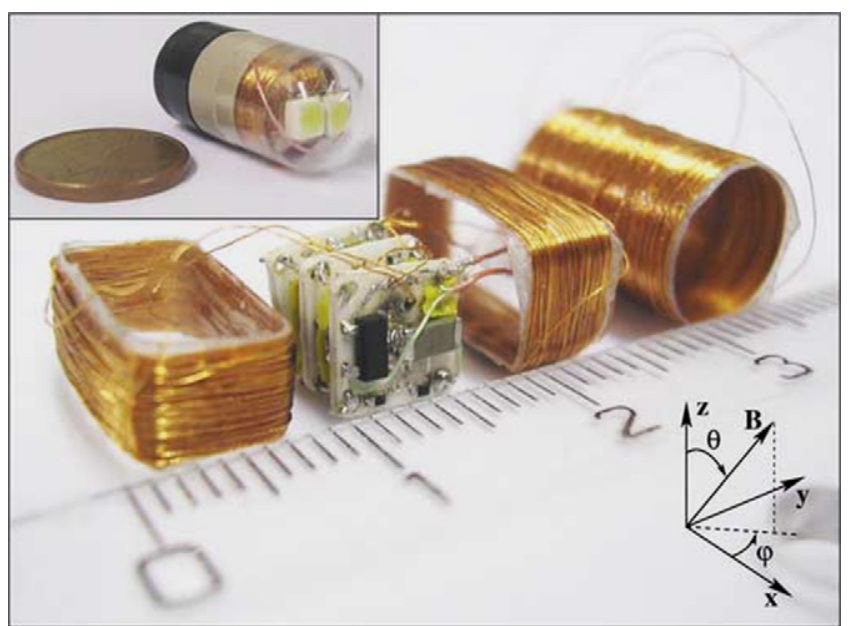

Fig. 2. The disassembled power receiver: three orthogonal coils and receiver electronics. Upper left: assembled with two white LEDs inside a biocompatible package, next to a 1-eurocent coin.

The parallel capacitance normally present in a class E inverter has been replaced here by a diode. The diode allows for suboptimum operation, i.e. a class C behaviour (Sokal and Sokal, 1975; Kazimierczuk and Czarkowski, 1995). This makes the circuit insensitive to variations in $R_{1}$, which would otherwise require to have a well specified value. Values of $R_{1}$ of up to $3 \Omega$ can be accommodated for. Parasitic capacitances compensate for the absence of the parallel capacitor, the exact value of which is not critical for suboptimum operation.

The driver was designed to deliver a maximum current amplitude of $11 \mathrm{~A}$ through the primary coil, corresponding to a voltage amplitude of $4 \mathrm{kV}$ over the tank. A $V_{\mathrm{CC}}$ of $120 \mathrm{~V}$ needs to be applied for this. As is shown further in this text, a maximum current of only $7 \mathrm{~A}$ is required to meet the power specifications. A power conversion efficiency of $90 \%$ is measured.

\subsection{Power receiver}

The power receiver comprises three coils which are geometrically orthogonal to one another, ensuring adequate coupling with the primary coil for any orientation of the capsule. Fig. 2 shows how the three orthogonal coils fit into one another and envelop the receiver electronics. The coils were wound out of full copper wire with a diameter of $100 \mu \mathrm{m}$. The orthogonal co-ordinate system in Fig. 2 indicates the sensitive axes of the coils. The $x$-axis for example corresponds to the cylindric coil while the $z$-axis corresponds to the smallest of the rectangular coils. The assembled receiver is a cylinder with outer dimensions of $\varnothing 10 \mathrm{~mm} \times 13 \mathrm{~mm}$. Two white LED's are connected to the $3.6 \mathrm{~V}$ output for demonstration purposes. The whole is concealed within a pill shaped package with an outer diameter of $11 \mathrm{~mm}$, also depicted in Fig. 2. The capsule package was machined out of materials that are biocompatible for wireless endoscopy by the IVP partners from SSSA Pisa, Italy. The materials used are peek, Delrin and Plexiglas.

In Fig. 3, the electric schematic of the receiver system is depicted. Resonance capacitors $C_{2 x, 2 y, 2 z}$ cancel the inductances 


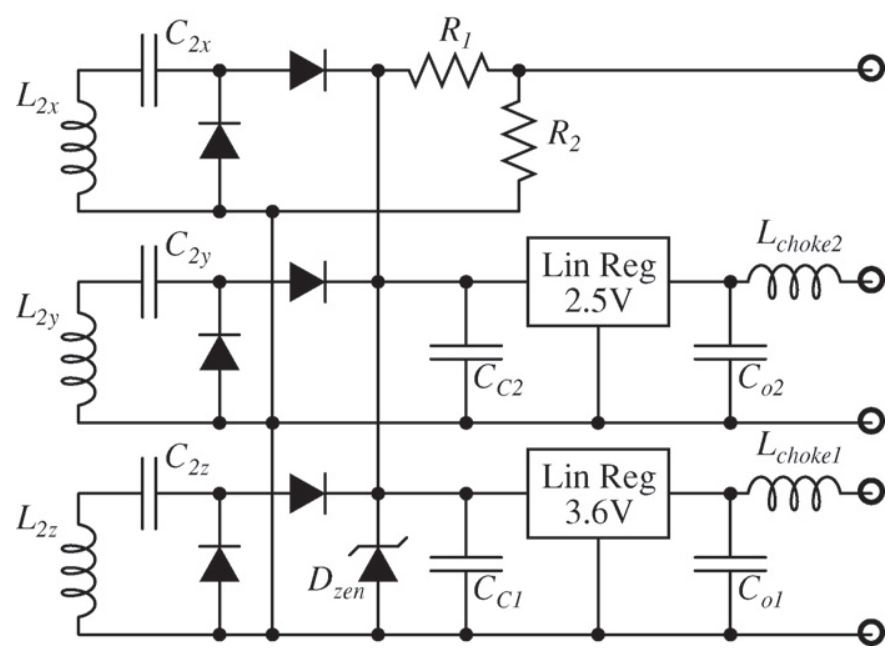

Fig. 3. The power receiver schematic.

of the secondary coils. The rectifiers force the positive part of their input current through the common collector capacitors $C_{\mathrm{C} 1}$ and $C_{\mathrm{C} 2}$. In this way, the contributions of all three rectifiers are summed. There is always at least one operational, because of the orthogonality of the receiver coils.

Two regulators are present, as two different supply voltages are required inside the capsule: one at $3.6 \mathrm{~V}$ and one at $2.5 \mathrm{~V}$. To protect the regulators from an eventual over-voltage, a zener diode $\mathrm{D}_{\text {zen }}$ is installed $\left(V_{\mathrm{z}}=5.6 \mathrm{~V}\right)$. The output capacitors $C_{\mathrm{o} 1}$ and $C_{\mathrm{o} 2}$ are required for output stability of the regulators. Together with $L_{\text {choke1 }}$ and $L_{\text {choke2 }}$ and additional decoupling capacitors which are to be placed near the load circuitry, they constitute a CLC-network. Besides from adding more low-pass filtering, this CLC-network suppresses interference from the omnipresent magnetic field.

The resistors $R_{1}$ and $R_{2}$ scale the unregulated dc voltage for onboard A/D conversion. This information is to be transmitted to the outside world over the capsule data link to adjust the input power at the external side for varying coupling conditions and the changing power request of the capsule.

Three ceramic substrates were designed and fabricated in order to realize the needed interconnections. An in-house double-sided thick-film screen printing technology was used. The finished substrates are stacked and are mechanically and electrically interconnected by four wires leading through four holes in their corners (Fig. 2).

\section{Link performance}

The power efficiency of the link prototype is measured. The efficiency $\eta$ is defined here as the ratio of the dc power delivered to the regulator, to the power dissipated by the external voltage source $V_{\mathrm{CC}}$. The losses in the regulator itself are not included. These are directly proportional to the voltage between regulator's input and output.

The power receiver is positioned in the center of the primary coil by means of a construction made out of PVC. The orientation of the receiver is adjustable. A methodology that is similar

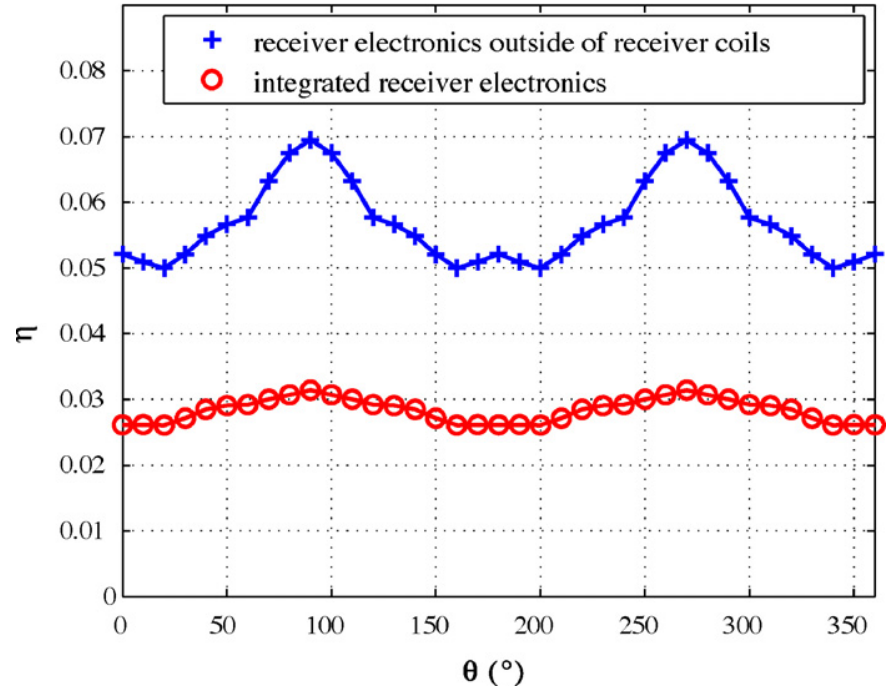

Fig. 4. Measured link efficiency with the receiver coils positioned in the center of the primary coil $(\varphi=0)$.

to the one described in preceding work (Lenaerts and Puers, 2005) is applied to measure the link efficiency. Fig. 4 shows the resulting efficiency data when the magnetic flux density vector $B$ is rotated in the $x z$-plane of the receiver (Fig. 2). The efficiency with the receiver electronics placed outside of the coil set is plotted together with the efficiency when the electronics are fully integrated inside the coils.

The minima and maxima in link efficiency are encountered at, or close to orientations of the magnetic field that coincide with one of the coil axes. This holds for orientations with $\varphi \neq 0$ as well. When the receiver coil that makes the best inductive link is oriented along the magnetic field vector, a maximum in efficiency is reached. For the coil set used here, this is the $x$ coil. The minimum in efficiency is found for a magnetic field orientation close to the $z$-direction (Lenaerts and Puers, 2005).

One can see from Fig. 4 that the worst-case efficiency of the link is roughly halved by integrating the receiver electronics. This is due to a decrease in quality factor of the receiver coils. The equivalent series resistance of the $z$-coil was found to double when it was placed around the substrate stack. The additional losses are caused by eddy currents induced in the metal parts of the electronic components. The performance of the link can thus be improved by decreasing the volume of metal in the receiver components. The chip components can be purchased in die form and wire-bonded directly to the ceramic carrier. This eliminates the metal used for packaging. The volume of capacitors and inductors can be reduced by lowering the filtering demands. For this prototype specifically, the big $1 \mathrm{kV} 1206$ resonance capacitors could be replaced by their $200 \mathrm{~V} 0805$ counterparts. This smaller size was not available to the authors at the time of realization.

For a worst-case orientation, the efficiency of the prototype is read to be $2.6 \%$ from Fig. 4 . This is for a capsule positioned in the exact center of the primary coil. In reality, the capsule will operate throughout the volume enclosed by the patient's small bowel and/or other regions of interest. As the strength of the 
magnetic field induced by the primary coil varies throughout space, there exists a point within the explored volume where the induced field is weakest. This point defines the worst-case position of the capsule, as the link efficiency will reach a lower limit here. If the capsule finds itself in this worst-case position as well as adopting a worst-case orientation, the overall worst-case efficiency is found.

A 2D axisymmetric finite element (FE) analysis was carried out to find the magnetic field strength distribution throughout the primary coil volume. From the result, a volume can be visualized that is delimited by a given field strength value. With a field strength value corresponding to a link efficiency of $1.1 \%$, always assuming worst-case capsule orientation, a volume is found that covers at least the small bowel volume (Supplementary Fig. 1). With typical regulator losses included, this comes down to an overall worst-case power efficiency of about $1 \%$, from the external dc supply to the remote load.

An efficiency of $1 \%$ implies a current of $7 \mathrm{~A}$ through the primary coil to reach the power specification of $150 \mathrm{~mW}$. However, the capsule will not find itself under worst-case conditions continuously and more typical working values will be around 4 A.

\section{Biological tissue interaction}

Thus far, the realized inductive link was only tested with air as the surrounding medium. Biological tissue is slightly conductive (Gabriel et al., 1996a,b) and will therefore draw power from an electromagnetic field. The power dissipation in the patient's body will impose a limitation on the primary coil current for reasons of health and safety. Furthermore, the performance of the link and the tuning of the class $\mathrm{E}$ driver will be affected.

\subsection{Specific absorption rate}

The specific absorption rate (SAR) is defined as the amount of power dissipated per unit mass. Modern RF exposure stan- dards are mostly expressed in terms of SAR. The International Commission on Non-Ionizing Radiation Protection (ICNIRP, 1998) sustains a SAR limit of $0.4 \mathrm{~W} / \mathrm{kg}$, averaged over the whole body. This is for continuous and occupational exposure, meaning that the subject is an informed, healthy adult.

In order to quantify the heat dissipation in the patient's body due to the induced magnetic field, the ESR $R_{1}$ of the primary coil is measured. The ESR is a direct measure for the dissipation in the coil windings and in parasitically coupled conductors, such as the patient's body. Hence, the whole body SAR can be calculated from the excess $R_{1}$ that is measured when introducing a human test subject. Since the frequency dependent ESR of a high-quality inductor is extremely hard to measure, a highquality capacitor is connected in parallel with the coil. A vector analyzer with S-parameter test set is used to observe the resonance of this network. From the height of the resonance peak, an accurate value for $R_{1}$ at that frequency is found. Eight different capacitances were used, yielding eight different frequencies. Three different coil set-ups were evaluated: an empty coil, a coil with a human subject holding his arms up and a coil with a human subject holding his arms down against the coil (Supplementary Fig. 2). It is implicitly assumed that field strengths are below the level where non-linear mechanisms come into play inside the biological tissue.

In Fig. 5 the resulting SAR is plotted for a primary coil current of $7 \mathrm{~A}$. For a frequency of $1 \mathrm{MHz}$, a whole-body SAR of $0.32 \mathrm{~W} / \mathrm{kg}$ is found in the arms up situation, and a SAR of $1.16 \mathrm{~W} / \mathrm{kg}$ in the arms down situation. The norms prescribed by the ICNIRP are thus exceeded in the latter case.

Fig. 5 also shows the measured tank impedance versus frequency for one of the LC combinations. A decrease in resonance frequency is consistently observed when biological tissue is brought closer to the windings. If the interaction with the tissue were to be purely inductive, an increase in resonance frequency was to be expected, as inductively coupled circuits decrease the effective inductance seen at the primary coil terminals. A decrease in resonance frequency can only be explained by a capacitive interaction which is predominant over the inductive

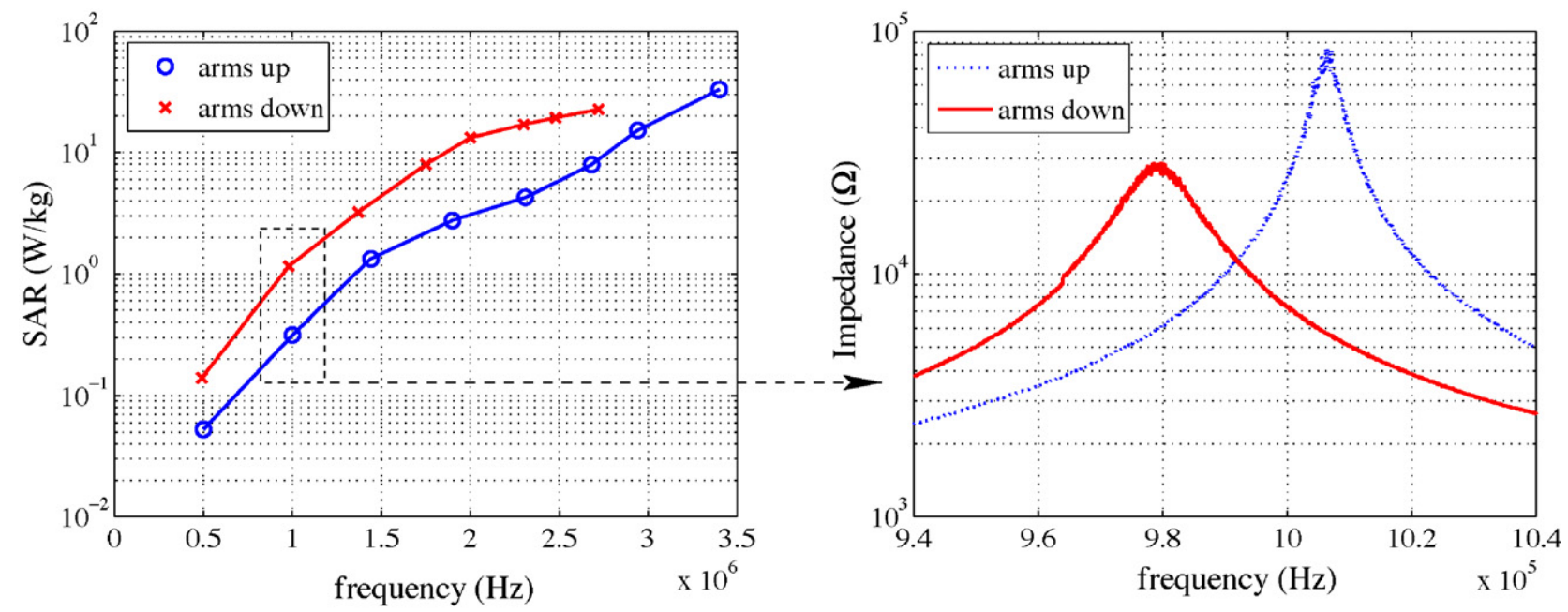

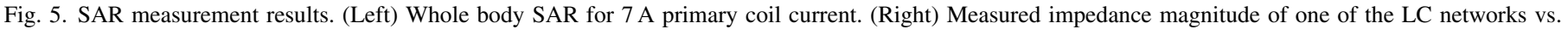
frequency. 

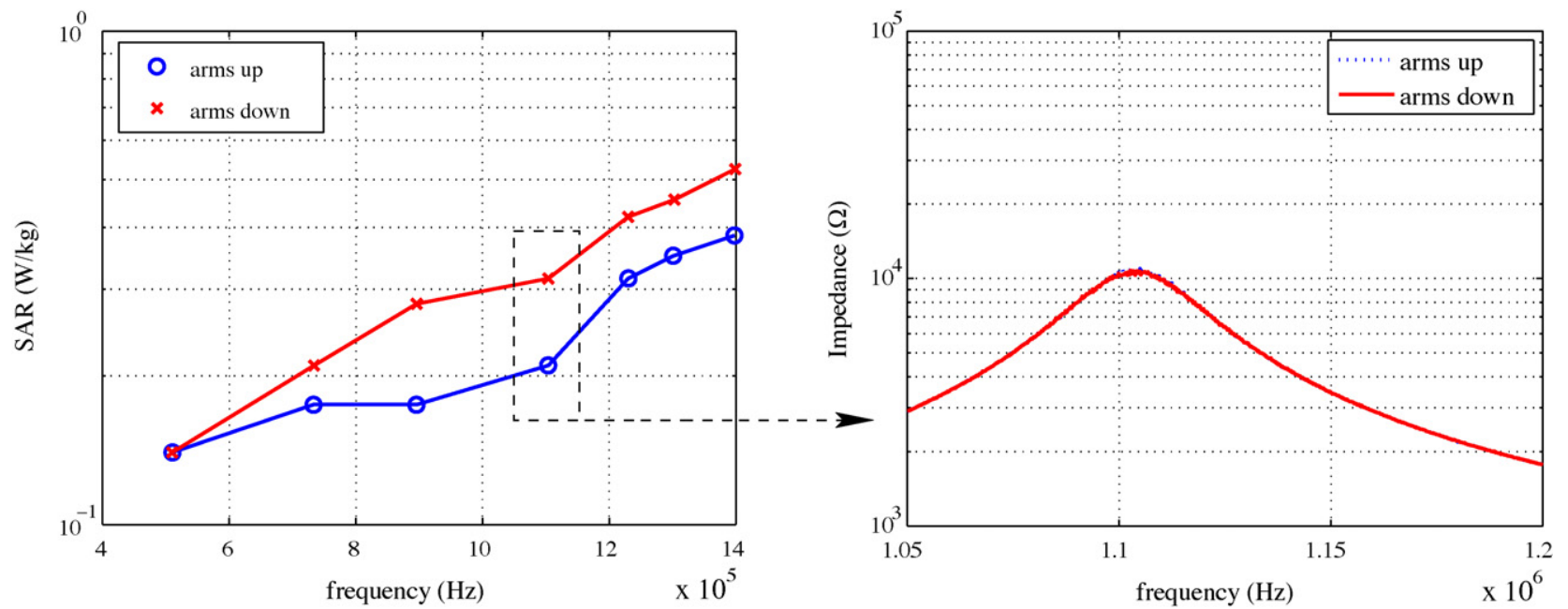

Fig. 6. SAR measurement results with an electrically shielded coil. (Left) Whole body SAR for 7 A primary coil current. (Right) Measured impedance magnitude of one of the LC networks vs. frequency.

one. The capacitive effect in question is caused by a conservative electric field. This field stems from the space charge distribution that goes together with the voltage maintained over the primary coil. The slightly conductive human body acts as a short circuit for these electric field lines and in this way effectively enhances the inter-winding capacitance.

It is possible to shield off penetrating electric fields by terminating the field lines on a conductive shield that is kept at a fixed potential. To this purpose, a copper foil is applied to the inner and outer surface of the primary coil and electrically connected to the zero potential terminal. A slid is spared out along the length of the coil to avoid making a parasitically coupled coil. On the outer surface only two patches of copper foil are applied there where the arms touch.

The same SAR measurements were repeated with the shielded solenoid. The results are plotted in Fig. 6. Adding the copper sheet lowers the self-resonance frequency of the coil to 1.4 MHz. Therefore, only frequencies up to $1.4 \mathrm{MHz}$ could be investigated. Comparing this plot with Fig. 5 points out that human body dissipation is greatly reduced by shielding off the conservative electric field, especially in the arms down situation. The SAR requirement of $0.4 \mathrm{~W} / \mathrm{kg}$ is now met for all postures of the patient at $1 \mathrm{MHz}$.

\subsection{Link performance}

Energy dissipation in parasitically coupled bodies, like the patient's body and the electric shield, decrease the efficiency of the link.

Although the magnetic field may be locally distorted by eddy current flow in the copper shield, the overall field strength remains unaffected within the bulk of the solenoid. Hence, the primary current amplitude does not need to be increased to reach the power specifications. The decrease in efficiency is reflected only by a change in phase difference between current through and voltage over the primary coil.

The eddy current losses in the electric shield may be reduced by exchanging the full copper foil for a net of thin conductors.
This net should not contain closed loops. A comb structure could be used for instance. Another approach is to use a lesser conductor than copper for the foil.

The dielectric losses in the parasitic capacitance constituted by the shield can be decreased by selecting a low-loss and/or lowpermittivity dielectric for the material in between coil windings and shield.

\subsection{Class E tuning}

As any resonance tuned circuit, the class E inverter is very sensitive to component tolerances. The circuit depicted in Fig. 1 yields a class $E$ behaviour (or class $C$, for suboptimum operation) at a pulse frequency of $1055 \mathrm{kHz}$. Deviations of only $5 \mathrm{kHz}$ on this value will bring the circuit out of class $\mathrm{E}$ regime, making the output waveforms to collapse. Conversely, a small deviation on component values will change the frequency at which class E behaviour occurs.

The presence of biological tissue does not only alter the equivalent series resistance of the primary coil, it also changes the reactive component of its impedance. Primary coil $L_{1}$ and capacitor $C_{\text {tank }}$ in Fig. 1 constitute a semiresonant tank. Fig. 5 shows the shift in resonance frequency that can be expected when the arms are moved between two extreme positions. These kind of variations are not supported and will make the output current to collapse.

As already discussed above, the biological tissue interaction is predominantly capacitive in nature. If electrical shielding is applied, this capacitive effect is eliminated. Fig. 6 shows an impedance measurement with the patient inside an electrically shielded coil. The resonance peaks for the two different arm positions now coincide perfectly. No difference is measurable. By introducing an electric shield, the class $E$ tuning has become insensitive to the patient's posture.

When big, metal objects are brought into the primary coil's vicinity, there is still a significant shift in tank resonance frequency observed. The same goes for mechanical deformation of the coil. In both cases, the effect is inductive and therefore 
unavoidable. If one needs to deal with metal bodies or with coil deformations, some kind of automatic tuning has to be applied. This is done for example through frequency control (Troyk and Schwan, 1992). The drawback of this technique are the large shifts in operation frequency. These drastically deteriorate the link efficiency, as the receiver is tuned to one particular frequency. Another option involves impedance compensation by means of a transductor (Schuylenbergh and Puers, 1996). A transductor is an inductor with an electrically controllable inductance value.

\section{Conclusion}

The position and orientation of a camera capsule within the abdominal volume are random and varying. In order to continuously capture high-quality images, a power of $150 \mathrm{~mW}$ is demanded at any time. An inductive power link that meets these requirements is presented. The receiver electronics are integrated inside the receiver coils to have the whole fit within a volume of $\varnothing 10 \mathrm{~mm} \times 13 \mathrm{~mm}$. The overall efficiency from external input to usable remote dc power is measured to be $1 \%$ under worst-case conditions. Issues regarding the interaction with biological tissue are reported. The appliance of an electric shield on the primary coil inner and outer surface greatly reduces the interaction. Thanks to electric shielding, the overall specific absorption rate (SAR) in the patient's body remains below the limit of $0.4 \mathrm{~W} / \mathrm{kg}$, as prescribed by the ICNIRP. Furthermore, the electric shield makes the tuning of the resonant coil driver insensitive to movements of the patient. In this way, a classic open-loop class E inverter can be used in appliance on a living subject.

\section{Acknowledgment}

The authors are grateful to the European Commission for the financial support of this work through the IVP IST-project
(IST-2001-35169). Special thanks goes out to Alberto Arena of SSSA, Pisa for providing the capsule package.

\section{Appendix A. Supplementary data}

Supplementary data associated with this article can be found, in the online version, at doi:10.1016/j.bios.2006.06.015.

\section{References}

Appleyard, M., Fireman, Z., Glukhovsky, A., Jacob, H., Shreiver, R., Kadirkamanathan, S., Lavy, A., Lewkowicz, S., Scapa, E., Shofti, R., Swain, P., Zaretsky, A., 2000. Gastroenterology 119 (6), 1431-1438.

Arena, A., Boulougoura, M., Chowdrey, H., Dario, P., Harendt, C., Irion, K.M., Kodogiannis, V., Lenaerts, B., Menciassi, A., Puers, R., Scherjon, C., Turgis, D., 2005. In: Bos, L., et al. (Eds.), Medical and Care Compunetics, vol. 2. IOS Press, pp. 167-174.

Costamagna, G., Shah, S.K., Riccioni, M.E., Foschia, F., Mutignani, M., Perri, V., Vecchioli, A., Brizi, M.G., Picciocchi, A., Marano, P., 2002. Gastroenterology 123 (4), 999-1005.

Fireman, Z., Mahajna, E., Broide, E., Shapiro, M., Fich, L., Sternberg, A., Kopelman, Y., Scapa, E., 2003. Gut 52, 390-392.

Gabriel, C., Gabriel, S., Corthout, E., 1996a. Phys. Med. Biol. 41, 22312249.

Gabriel, S., Lau, R.W., Gabriel, C., 1996b. Phys. Med. Biol. 41, 2251-2269.

Iddan, G., Meron, G., Glukhovsky, A., Swain, P., 2000. Nature 405, 417.

International Commission on Non-Ionizing Radiation Protection, 1998. Health Phys. 74 (4), 494-522.

Kazimierczuk, M.K., Czarkowski, D., 1995. Resonant Power Converters. John Wiley \& Sons, New York, pp. 347-378.

Ko, W.H., Liang, S.P., Fung, C.D.F., 1977. Med. Biol. Eng. Comp. 15, 634-640. Lenaerts, B., Puers, R., 2005. Sens. Actuators A 123-124, 522-530.

Puers, R., Catrysse, M., Vandevoorde, G., Collier, R., Louridas, E., Burny, F., Donkewolcke, M., Moulart, F., 2000. In: Eiler, J., Alcorn, D., Neuman, M. (Eds.), Biotelemetry XV, Proceedings 15th ISOB Conference on Biotelemetry. Thomson-Shore Inc., Michigan, USA, pp. 652-660.

Raab, F., 1977. IEEE Trans. Circuits Syst. CAS-24, 725-735.

Schuylenbergh, K.V., Puers, R., 1996. Sens. Actuators A 52, 1-7.

Sokal, N.O., Sokal, A.D., 1975. IEEE J. Solid-State Circuits SC-10, 168-176. Sullivan, C.R., 1999. IEEE Trans. Power Electron. 14, 283-291.

Troyk, P.R., Schwan, A.K., 1992. IEEE Trans. Biol. Eng. 39 (6), 589-599. 